\title{
Development of Year 2020 Goals for the National HIV/AIDS Strategy for the United States
}

\author{
David R. Holtgrave
}

Published online: 11 August 2013

(C) Springer Science+Business Media New York 2013

\begin{abstract}
In July, 2010, President Barack Obama released the National HIV/AIDS Strategy (NHAS). The NHAS set forth ambitious goals for the year 2015. These goals were potentially achievable had the appropriate level of resources been invested; however, investment at the necessary scale has not been made and the 2015 goals now may well be out of reach. Therefore, we propose that an updated NHAS be developed with goals for the year 2020 clearly articulated. For the purposes of fostering discussion on this important topic, we propose bold yet achievable quantitative 2020 goals based on previously published economic and mathematical modeling analyses.
\end{abstract}

Keywords HIV · Policy · Mathematical modeling · National HIV/AIDS Strategy $\cdot$ Economics

In July 2010, President Barack Obama released the National HIV/AIDS Strategy (NHAS) [1]. The landmark strategy contained ambitious but achievable goals for the year 2015; these goals are listed in Table 1 (quoted exactly as they appear in the NHAS). The goals spanned the areas of HIV prevention, care, reduction of disparities, and improved coordination of HIV/AIDS efforts programs and policies. In his speech launching the NHAS, the President stated: "So the question is not whether we know what to do, but whether we will do it. Whether we will fulfill those obligations; whether we will marshal our resources and the political will to confront a tragedy that is preventable [2]."

D. R. Holtgrave $(\varangle)$

Department of Health, Behavior and Society, Johns Hopkins

Bloomberg School of Public Health, 624 N Broadway,

Baltimore, MD 21205, USA

e-mail: dholtgra@jhsph.edu
The NHAS makes clear that strategic HIV prevention, care and housing programs and policies implemented sooner rather than later can have a profound and lasting impact on the epidemic $[1,2]$.

While the NHAS spelled out critical goals to be achieved in 5 years, and gave considerable detail as to the services and policies that needed to be in place to achieve those goals, it was silent on the issue of the level of resources needed to achieve the goals [1, 3, 4]. Therefore, in 2010, we estimated the costs necessary to achieve the NHAS goals, and used mathematical modeling to estimate the effectiveness and cost-effectiveness of making such an investment [3].

However, the ensuing years saw roughly level funding for HIV-related programs in the US overall, with most programmatic areas being relatively flat-funded, or receiving small percentage increases well below the amount estimated as necessary to achieve the NHAS goals $[3,4]$. Some jurisdictions even found their federal HIV funding reduced substantially for certain programs. Therefore, in 2012 we conducted an updated analysis to determine if it was still possible to achieve the 2015 NHAS goals. We found that if a large and rapid scale up of domestic HIV diagnostic, care, housing and prevention services for persons living with HIV were made in 2014 and 2015 (with a combined 2-year increased investment of roughly $\$ 17$ billion across both the public and private sectors where the additional resources might be new or strategically redirected), then the NHAS goals of $25 \%$ reduction in incidence and $30 \%$ reduction in transmission rate could still be achieved. However, if the scale up does not begin in earnest in 2014, then the goals will most likely not be achieved as a 1-year effort in 2015 would be too late to scale up programs and achieve the large effects necessary all within the same year [4]. 
Table 1 Major 2015 goals of the National HIV/AIDS Strategy issued in 2010

\section{Reducing new HIV infections ${ }^{\mathrm{a}}$ \\ Lower the annual number of new infections by $25 \%$; \\ Reduce the HIV transmission rate, which is a measure of annual transmissions in relation to the number of people living with HIV, by $30 \%$; and, \\ Increase from 79 to $90 \%$ the percentage of people living with HIV who know their serostatus. \\ Increasing access to care and improving health outcomes for people living with HIV $^{\mathrm{a}}$ \\ Increase the proportion of newly diagnosed patients linked to clinical care within 3 months of their HIV diagnosis from 65 to $85 \%$; \\ Increase the proportion of Ryan White HIV/AIDS program clients who are in continuous care (at least 2 visits for routine HIV medical care in 12 months at least 3 months apart) from 73 to $80 \%$; and \\ Increase the percentage of Ryan White HIV/AIDS program clients with permanent housing from 82 to $86 \%$. (This serves as a measurable proxy of our efforts to expand access to HUD and other housing supports to all needy people living with HIV.) \\ Reducing HIV-related disparities and health inequities ${ }^{\mathrm{a}}$ \\ Increase the proportion of HIV diagnosed gay and bisexual men with undetectable viral load by $20 \%$; \\ Increase the proportion of HIV diagnosed Blacks with undetectable viral load by $20 \%$; and, \\ Increase the proportion of HIV diagnosed Latinos with undetectable viral load by $20 \%$. \\ Achieving a more coordinated national response to the HIV epidemic $^{\mathrm{a}}$ \\ Increase the coordination of HIV programs across the Federal Government and between Federal agencies and State, territorial, local, and tribal governments; and, \\ Develop improved mechanisms to monitor and report on progress toward achieving national goals.}

${ }^{a}$ All section headings and all goals are quoted here exactly as presented in the federal National HIV/AIDS Strategy issued in July 2010 [1]; quotation marks around all items have been suppressed for ease of presentation

Sadly, that fiscal year 2014 scale up appears highly unlikely to occur. The overall economic climate has made private sector investment in HIV/AIDS efforts more difficult. Further, governmental spending has been challenged. In fiscal year 2012, the total US federal investment in domestic HIV programs was about \$21.4 billion [5]. In the President's fiscal year 2014 budget request to Congress, he asked for $\$ 23.2$ billion [5, 6]. However, the federal sequester is now lessening the current fiscal year 2013 funding available to HIV programs [7], and the fiscal year budget outlook in Congress for fiscal year 2014 would seem to indicate that one or more continuing resolutions (indicating rather level funding with the impact of a 2nd year of sequestration incorporated) appears to be the most likely appropriations outcome [8]. Hence, while the
President requested a fraction of the increased resources needed for domestic HIV programs, even this amount is very unlikely to be supported in the Congressional budgeting process. We know of no scenario that indicates that the necessary level of scale up of domestic HIV programs for achieving the 2015 NHAS goals will begin in 2014.

Therefore, achievement of the 2015 NHAS goals is in serious jeopardy. This is extremely unfortunate because failure to achieve the goals portends serious, negative morbidity, mortality and economic consequences [3, 4]. The question then becomes one of whether we should give up on the 2015 NHAS goals and abandon the NHAS. I argue that while the 2015 NHAS goals are now likely out of reach due to insufficient investment in the necessary programs at the requisite scale, the NHAS is an essential guidepost for achieving real and lasting change in the HIV epidemic in the US. Therefore, I assert that rather than abandon the NHAS, we should stretch the time horizon and increase the boldness of the goals in the hopes that over the coming years, the requisite investments will be made and "make up" for time lost in the current federal budget woes.

I propose that in 2014, a new NHAS be formulated with goals set for the year 2020. This reformulation should not simply push the current goals 5 years down the road, but rather should be informed by policy analyses to determine what goals might realistically be achievable by 2020 if there were sufficient investment made to scale up the NHAS. To be more specific, our recently published analysis of the costs and consequences of the NHAS provides some guidance as to what these goals might look like [4]. The proposed NHAS 2020 goals are given in Table 2.

We retain the four major segments of the NHAS (reducing HIV incidence, improving access to care, reducing health disparities, and improving service coordination), but note that the sections are very intertwined and synergistic with each other so the section differentiation is for ease of exposition than for imposing an artificial distinction between, say, prevention and care. Below, we update the goals in each section and discuss these in turn below, section by section. Further, we discuss a measurement strategy for each goal and assert that an annual report on the interim measurement of all goals should be accomplished and published.

\section{Goals Related to Reducing New Infections}

In our 2012 analysis of the costs and consequences of the NHAS, we found that it would be possible to reduce HIV incidence by $45.8 \%$ and reduce the transmission rate by $51.1 \%$ with sufficient scale up of diagnostic, care, housing and prevention services [4]. Therefore, we assert that a reasonable, modeling-based goal would be a reduction in 
Table 2 Proposed 2020 goals for a new National HIV/AIDS Strategy for the United States

\section{Reducing new HIV infections ${ }^{\mathrm{a}}$ \\ Lower the annual number of new HIV infections by at least $45 \%$ (relative to baseline year 2010); \\ Reduce the HIV transmission rate, which is a measure of annual transmissions in relation to the number of people living with HIV, by at least $50 \%$ (relative to baseline year 2010); \\ Increase to at least $90 \%$ the percentage of people living with HIV who know their serostatus (with an emphasis on identifying seropositivity as soon as possible after HIV infection); and,}

Further reduce the already low number of diagnosed persons living with HIV who engage in unprotected, serodiscordant, transmission-relevant risk behavior by at least $50 \%$ (relative to baseline year 2010).

Measurement: all four quantitative constructs above are now estimated and published by CDC [9-12].

Increasing access to care and improving health outcomes for people living with $\mathrm{HIV}^{\mathrm{a}}$

Ensure that at least $85 \%$ of newly diagnosed patients living with HIV are linked to clinical care within 3 months of their HIV diagnosis, and that at least $85 \%$ of all diagnosed persons living with HIV are retained in care;

Ensure that at least $81 \%$ of clients receiving HIV care achieve and maintain viral suppression; and,

Ensure that at least $90 \%$ of persons living with HIV in need of stable housing services receive and retain such services.

Measurement: the first and second goals in this section can be measured by existing CDC and HRSA systems [9-16]. In the 2010 NHAS, the housing goal referenced Ryan White clients; we propose a broader measurement strategy reflecting more persons living with HIV (such as an expansion of CDCs Medical Monitoring Project [13, 15]).

Reducing HIV-related disparities and health inequities ${ }^{\mathrm{a}}$

Ensure that all goals listed in the care section and the seropositivity awareness goal are all achieved for all genders, racial/ethnic groups, sexual minorities, heightened risk groups (such as persons who inject drugs), and age groups;

Among the nine subpopulations defined by CDC as totaling more than $85 \%$ of the HIV incidence in the US in 2010, ensure that no subpopulation has more than 2,550 new HIV infections per year (and that incidence is level or decreasing for all subpopulations in the US in all years); and,

Develop and annually report on measures designed to assess HIV-related stigma experienced by persons living with HIV (especially, to gauge how such experiences serve as barriers to entry into, or retention in, HIV care).

Measurement: the first goal in the disparities section can be measured by seemingly feasible subgroup analyses in CDC and HRSA systems [9$14,16]$. The second goal can be measured from subgroup analyses now conducted and published by CDC [12]. The third disparities goal is a directly-observable, nominal-scale process goal.

Achieving a more coordinated national response to the HIV epidemic ${ }^{\mathrm{a}}$

Continue efforts to constantly refine and annually report upon the coordination of HIV programs across the Federal Government and between Federal agencies and State, territorial, local, and tribal governments; and,

Continually refine and annually report on metrics necessary to monitor and react to progress toward achieving all national goals.

Measurement: the goals in this section are directly-observable, nominalscale process goals. The second coordination goal builds upon existing efforts by the Department of Health and Human Services to have all HIV federally-funded service delivery programs monitor a small, core set of NHAS-relevant core indicators [15].

${ }^{a}$ All four section headings are quoted exactly as presented in the federal National HIV/AIDS Strategy issued in July 2010 [1]; goals in each section are newly proposed or modified here incidence of at least $45 \%$ and a reduction in transmission rate of at least $50 \%$ by 2020 . Throughout, we use the modifier "at least" to indicate that more ambitious goals are clearly desirable and that more intensive investment could provide even more impact. While we maintain the original goal of $90 \%$ awareness of HIV seropositivity, we modify it to say "at least" $90 \%$ and include a parenthetical phrase to emphasize the importance of early diagnosis.

We add a new goal to this section to further reduce unprotected serodiscordant risk behavior by diagnosed persons living with HIV that could result in HIV transmission from an already low level baseline of roughly $16 \%$ [4]. We added this goal because prior analyses have shown that prevention services for persons living with HIV engaged in unprotected serodiscordant risk behavior can be effective, cost-effective and have an important impact on HIV transmission in the US [3, 4, 17]. Such "prevention with positives" services were highlighted in the NHAS, but no specific goal was given [1, 4].

Clearly, all proposed goals should be measurable, and in Table 2, we provide the references to support the assertion that the US Centers for Disease Control and Prevention (CDC) currently estimates and publishes all of the statistics related to the proposed goals in this section [9-12]. Therefore, the roots of the proposed goals in this section have origins in previously published mathematical modeling and economic evaluation work, and are measurable under current CDC data collection and analytic systems.

\section{Access to Care Goals}

The original access to care section goals for 2015 relied heavily on achievement among Ryan White service clients. While we believe that it is very important to serve clients in Ryan White funded programs, in our modeling we sought to expand this notion of linkage to and retention in care for all diagnosed persons living with HIV [4]. Therefore, in Table 2, we proposed that there be at bare minimum $85 \%$ early linkage to care and at least $85 \%$ retention in care. This $85 \%$ level was assumed in our previous modeling [4]. It is tempting and highly desirable to aspire to a goal even higher than $85 \%$ for care linkage and for care retention given the tremendous promise that comes with the advent of the Affordable Care Act (ACA) in the US. However we recommend $85 \%$ as a minimal goal due to concerns about some states choosing not to expand Medicaid as allowed under the ACA and some key ACA provisions related to employer health insurance coverage being delayed [18]. These decisions and delays may pose critical challenges for some persons living with HIV attempting to get quality care, so going above $85 \%$ sadly may not be feasible. The proposed linkage and retention goals are measurable with 
current CDC and HRSA measurement and evaluation systems [9-16].

In the care section, we reassert a goal related to housing, but change it in two ways. First, the original housing goal related to Ryan White clients and aspired to improve the $82 \%$ coverage of housing services to $86 \%$ of Ryan White clients needing them [1]. While we used that goal level in previous economic evaluation work [4], we believe an NHAS revision is the perfect time to move that low-aspiration goal to a more ambitious posture (attempting to achieve $90 \%$ coverage of housing services at a minimum) since HIV-related housing needs are so severe in the US [19]. Also, we propose expanding it from Ryan White clients to all persons living with HIV who may need stable housing services. CDCs Medical Monitoring Project (MMP) aims to be a representative sample of all persons living with HIV in care in the US and could be a useful vehicle for measuring levels of homelessness and unstable housing among persons living with HIV. However, it is important to note that MMP in its current form will not adequately measure housing needs among people living with HIV who are not engaged in HIV care $[5,13]$.

Also in the care section, we propose a new goal: that at least $81 \%$ of persons living with HIV who are in care, achieve suppressed viral load. In prior mathematical modeling, we assumed this value to be between 69.4 and $80.7 \%$ based on CDC data [4]. CDC recently published a detailed analysis breaking out viral suppression percentages among various subgroups of persons living with HIV, dividing the analyses along the lines of gender, race/ethnicity, transmission category, and age [16]. A very nearly $80 \%$ level of viral suppression among persons retained in care was found for persons living with HIV who are 55-64 years old (across all other demographic factors) and this level was higher than for other demographic categories; hence, we use this as $81 \%$ goal level as one already essentially achieved for one demographic group, but which is aspirational for serving other demographic groups of persons living with HIV. Therefore, this goal is rooted in evidence and in previous modeling exercises, and yet aspirational for some subgroups of persons living with HIV; moreover, this goal construction is useful for serving to address key issues of health disparities.

\section{Reducing Health Disparities}

In the original NHAS section on reduction of HIV-related health disparities, there was much critical discussion about the severely negative impacts that racism, homophobia, discrimination and HIV related stigma have on the HIV epidemic in the US [1]. However, the quantitative goals for that section of the NHAS all had to do with reduction of viral load among gay and bisexual men, black men and women, and Latino/Latina communities.

Here, we propose a somewhat different configuration. First, we propose a goal that asserts that all of the caresection goals and the seropositivity awareness goal from the incidence reduction section should be met for all persons living with (and affected by) HIV. This would mean that all of these goals would be met for all persons regardless of gender, race/ethnicity, sexual orientation, transmission risk, and age. We believe this is a more comprehensive approach to ensure the reduction of health disparities for goals which are focused on achievement of a certain, absolute level of service delivery coverage or on achievement of a certain, absolute outcome level. It includes, but clearly is not limited to an outcome of viral load reduction. It appears that CDC and HRSAs data collection and analysis systems could provide the necessary subgroup analyses required to monitor this goal [916].

The first goal of the disparities section, however, does not include mention of goals from the incidence reduction section which are focused on relative percentage decreases in HIV incidence, transmission rate, and unprotected serodiscordant risk behavior reductions. To say that such goals should be achieved for all subpopulations would serve to freeze in place extant disparities. Therefore, in the health disparities section, we include a second goal which is designed to alleviate the heavy disproportionate burden of HIV infection among nine key populations identified by CDC as accounting for over $85 \%$ of the HIV incidence in the US in 2010 [12]. According to CDC, these nine most heavily disproportionately impacted populations are as follows (with 2010 incidence given in parentheses): white men who have sex with men (MSM) $(11,200)$; black MSM (10,600); Latino MSM (6,700); black heterosexual women $(5,300)$; black heterosexual men $(2,700)$; white heterosexual women $(1,300)$; Latina heterosexual women $(1,200)$; black men who inject drugs $(1,100)$; and black women who inject drugs (850) [12]. The highest incidence among these subgroups is over 13 times higher than for the lowest incidence subgroup $(11,200 / 850)$; if we aimed to reduce that ratio from the baseline of over 13 to no more than 3 , it would mean that no subpopulation would have HIV incidence over 2,550 in a given year (3 times 850). One must insure this ratio of 3 is not achieved by having the lower, denominator value (850) increase; rather the ratio must be lowered while at the same time insuring that all subpopulations have level or decreasing incidence. If this second health disparities goal were achieved, then compared to 2010 baseline, then at least 23,750 infections would be averted (from a total of 47,500 for 2010) and our overall $45 \%$ incidence reduction goal would be achieved as well. 
We did not construct a similar health disparities goal for HIV transmission rate reduction because it is unclear that subgroup analyses could be conducted in any practical way due lack of information about HIV transmission frequency across subpopulations [4, 9]. Further, we did not construct a similar health disparities goal for unprotected serodiscordant risk behavior both because of measurement challenges as well as indications that for at least some populations there is no evidence of subpopulation differences (e.g., there seems to be little difference in levels of risk behavior among white and black gay men) [20, 21].

Also, we assert that a goal or goals should be added that address(es) the important but admittedly harder to quantify and measure social determinants of health such as racism, homophobia, stigma, and discrimination (something not done in the original NHAS goals). Given that there has been substantial recent work on the measurement of HIVrelated stigma [22, 23], we would propose at minimum a process goal that calls for the refinement, use and annual reporting on HIV-related stigma measures (with a special emphasis on how HIV-related stigma serves as a barrier to HIV care engagement at individual-, community-, and societal-levels). We believe that much further discussion is also needed on how to monitor and react to other key social determinants of HIV incidence and engagement in care. The Department of Health and Human Services' Office for Civil Rights and the Department of Justice could potentially play increased and key roles in fostering these important discussions (including the addressing of antiquated HIV criminalization statutes).

\section{Coordination Goals}

The original service and policy coordination goals were not quantitative in nature, and would indeed be difficult to quantify. Therefore, here we simply update the goals to (a) reflect recent work done by the Department of Health and Human Services on HIV service and policy coordination (such as convening the diverse HIV/AIDS Indictors Implementation Group with federal and non-federal partners to help develop, implement and monitor a core set of seven key, NHAS-relevant indicators approved by the HHS Secretary, and thereby beginning to devise mechanisms to strategically streamline and reduce the arduous reporting burden now placed on jurisdictions [15]); and (b) express the goals as easily-assessed, nominal-scale process measures. While the coordination of HIV services is a topic of central importance, it is not directly informed by our previous modeling efforts and so we give relatively less attention to the topic of coordination here. However, we would briefly note that some extremely important efforts to coordinate services suffer from the same problem of lack of investment as does the NHAS overall. For instance, on the third anniversary of the NHAS, President Obama signed an Executive Order establishing the HIV Care Continuum Initiative [24]. This federal working group has the critical task of striving to "coordinate Federal efforts to improve outcomes nationally across the HIV care continuum."[24] Unfortunately, this key Executive Order, too, is silent on the issue of resources necessary to address unmet needs.

In summary, we propose these goals not as a definitive articulation of their final form, but as a way to foster the beginning of a discussion of the important topics of (a) whether we should now stretch the time horizon of the NHAS given a level of investment sadly less than necessary to scale up services to needed levels to achieve the original goals by 2015; and (b) what evidence-based, achievable, yet assertive goals might look like and how they could be measured. It is unfortunate we are in a position to have to contemplate that the NHAS 2015 goals might not be realized; but failure to sufficiently invest in achievement of those goals should not cause use to abandon the general directions of the NHAS and it should not cause us to lose our aspiration to boldly change the course of the HIV epidemic in the US. The stakes are far too high.

Acknowledgments There was no Grant support specifically devoted to preparation of this manuscript.

Conflict of interest There are no other conflicts to declare.

\section{References}

1. The White House: National HIV/AIDS Strategy for the United States. July 2010. Online document available at http:// www.whitehouse.gov/sites/default/files/uploads/NHAS.pdf (2010). Accessed 19 July 2013.

2. President Barack Obama: Remarks by the President on the National HIV/AIDS Strategy. Online document available at http:// www.whitehouse.gov/the-press-office/remarks-president-nationalhivaids-strategy (2013). Accessed 19 July 2013.

3. Holtgrave DR. On the epidemiologic and economic importance of the National AIDS Strategy for the United States. J Acquir Immune Defic Syndr. 2010;55(2):139-42.

4. Holtgrave DR, Hall HI, Wehrmeyer L, Maulsby C. Costs, consequences and feasibility of strategies for achieving the goals of the National HIV/AIDS Strategy in the United States: a closing window for success? AIDS Behav. 2012;16(6):1365-72.

5. Kaiser Family Foundation: U.S. Federal Funding for HIV/AIDS: The President's FY 2014 budget request. Online document available at http://kff.org/hivaids/fact-sheet/u-s-federal-fundingfor-hivaids-the-presidents-fy-2014-budget-request/ (2013). Accessed 19 Jul 2013.

6. White House Office of Management and Budget: Budget of the United States Government, fiscal year 2014. Online document available at http://www.whitehouse.gov/omb/budget/Overview (2013). Accessed 19 July 2013.

7. National Alliance of State and Territorial AIDS Directors: The impact of budget sequestration on federal funding for state HIV/ 
AIDS and viral hepatitis programs. Online document available at http://nastad.org/docs/NASTAD-Fact-Sheet-PLA-SequestrationMay-2013.pdf (2013). Accessed 19 July 2013.

8. FCW. A season of gridlock. Online document available at http:// fcw.com/articles/2013/07/16/budget-outlook.aspx (2013). Accessed 19 July 2013.

9. Hall HI, Holtgrave DR, Tang T, Rhodes P. HIV transmission in the United States: considerations of viral load, risk behavior, and health disparities. AIDS Behav. 2013;17(5):1632-6.

10. CDC. Monitoring selected national HIV prevention and care objectives by using HIV surveillance data-United States and 6 U.S. dependent areas-2010. HIV surveillance supplemental report 2012;17(No. 3, part A):Table 5a (p. 22).

11. Freedman M, Mattson C, Johnson C, et al. Medical Monitoring Project: 2009-2010. Nationally representative estimates of sexual risk behavior among HIV+adults receiving medical care: US. Presented at 19th conference on retroviruses and opportunistic infections (poster abstract 1090), Seattle, WA, 5-8 Mar 2012.

12. CDC Fact Sheet: New HIV infections in the United States. Online document available at http://www.cdc.gov/nchhstp/newsroom/ docs/2012/HIV-Infections-2007-2010.pdf (2013). Accessed 19 July 2013.

13. CDC: Medical Monitoring Project (MMP). Online document available at http://www.cdc.gov/hiv/prevention/ongoing/mmp/ general.html (2013). Accessed 19 July 2013.

14. HRSA: 2010 State Profiles; Ryan White HIV/AIDS Program. Online document available at http://hab.hrsa.gov/stateprofiles/ index.htm (2013). Accessed 19 July 2013.

15. Department of Health and Human Services: Secretary Sebelius approves indicators for monitoring HHS-Funded HIV services. Online document available at http://blog.aids.gov/2012/08/ secretary-sebelius-approves-indicators-for-monitoring-hhs-fundedhiv-services.html (2013). Accessed 19 July 2013.

16. Hall HI, Frazier EL, Rhodes P, Holtgrave DR, Furlow-Parmley C, Tang T, Gray KM, Cohen SM, Mermin J, Skarbinski J. Differences in human immunodeficiency virus care and treatment among subpopulations in the United States. JAMA Intern Med. 2013;. doi:10.1001/jamainternmed.2013.6841.

17. Holtgrave DR, Maulsby C, Wehrmeyer L, Hall HI. Behavioral factors in assessing impact of HIV treatment as prevention. AIDS Behav. 2012;16(5):1085-91.

18. HIV Health Reform (AIDS Foundation of Chicago): State resources for ACA implementation. Online document available at http://www.hivhealthreform.org/state-resources-for-aca-implement ation/ (2013). Accessed 19 July 2013.

19. National AIDS Housing Coalition: HOPWA 2014 budget request: NAHC recommends $\$ 365.2$ million. Online document available at http://www.nationalaidshousing.org/PDF/2014HOPWANeed Paper (2013). Accessed 19 July 2013.

20. Maulsby C, Millett G, Lindsey K, Kelley R, Johnson K, Montoya D, Holtgrave D. A systematic review of HIV interventions for black men who have sex with men (MSM). BMC Public Health. 2013;13(1):625.

21. Maulsby C, Millett G, Lindsey K, Kelley R, Johnson K, Montoya D, Holtgrave D. HIV among black men who have sex with men (MSM) in the United States: a review of the literature. AIDS Behav. 2013. doi:10.1007/s10461-013-0476-2.

22. Kim JJ, Positive Charge Intervention Team, Maulsby C, Kinsky S, Holtgrave D. Understanding barriers to linking persons living with HIV to care in the United States: findings from the Positive Charge Initiative. Poster presented at the 2012 National Summit on HIV and Viral Hepatitis Diagnosis, Prevention and Access to Care, Washington, DC, 27 Nov 2012.

23. The People Living with HIV Stigma Index Partnership: The people living with HIV stigma index: user guide. Online document available at ttp://www.stigmaindex.org/90/publications/thepeople-living-with-hiv-stigma-index-user-guide.html (2013). Accessed 19 July 2013.

24. The White House: Executive Order-HIV Care Continuum Initiative. July 15, 2013. Online document available at http://www. whitehouse.gov/the-press-office/2013/07/15/executive-order-hivcare-continuum-initiative (2013). Accessed 23 July 2013. 